This document is the Accepted Manuscript version of a Published Work that appeared in final form in Analytical Chemistry, copyright (C) American Chemical Society after peer review and technical editing by the publisher. To access the final edited and published work see http://pubs.acs.org/doi/abs/10.1021/ac503069g 


\title{
Accurate Determination Of The Diffusion Coefficient Of Proteins By Fast Fourier Transformation With Whole Column Imaging Detection
}

\author{
Atefeh S. Zarabadi, Janusz \\ Pawliszyn* \\ Department Of Chemistry, University Of Waterloo, Waterloo, Ontario, Canada N2l 3G1 \\ E-mail: janusz@uwaterloo.ca
}

\begin{abstract}
Analysis in the frequency domain is considered a powerful tool to elicit precise information from spectroscopic signals. In this study, the fast Fourier transformation technique is employed to determine the diffusion coefficient (D) of a number of proteins in the frequency domain. Analytical approaches are investigated for determination of D from both experimental and data treatment viewpoints. The diffusion process is modeled to calculate diffusion coefficients based on the fast Fourier transformation solution to Fick's law equation, and its results are compared to time domain results. The simulations characterize optimum spatial and temporal conditions and demonstrate the noise tolerance of the method. The proposed model is validated by its application towards the electropherograms from the diffusion path of a set of proteins. Real time dynamic scanning is conducted by employing whole column imaging detection technology in combination with capillary isoelectric focusing (CIEF) and the imaging plug flow (iPF) experiment. These experimental techniques provide different peak shapes, which are

\footnotetext{
* To whom correspondence should be addressed
} 
utilized to demonstrate the fast Fourier transformation ability in extracting diffusion coefficients out of irregular shape signals. Experimental results confirmed that the fast Fourier transformation procedure enhanced the accuracy of the determined values up to $25 \%$ compared to those obtained in the time domain.

\section{Introduction}

The accurate measurement of the diffusion coefficient as an important physicochemical property of proteins has been extensively studied. Taylor and Aris were pioneers in the development of dispersing approaches, wherein a solute plug flows through an open tube, and $\mathrm{D}$ is calculated as a function of the solute diffusivity. ${ }^{1,2}$ Capillary zone electrophoresis was employed by Bello et al. for the fast measurement of the diffusion coefficients of both small and large molecules. ${ }^{3}$ However, the determination of diffusion coefficients using capillary electrophoresis is generally affected by variances in injection and detection. In order to reduce the influence of such variances on the calculation of $\mathrm{D}$, the "stopped migration" technique can be used; ${ }^{4}$ however, such a technique has a lengthy run-time. In order to address this issue, Jin and Chen developed a peak-height method that succeeded in shortening the measurement time while eliminating the variances effect. ${ }^{5}$ The unsteady-state flow caused due to the injection and pumping of solutes in capillary electrophoresis instrument has been corrected by Sharma et al. via modification to the Taylor analysis. ${ }^{6}$ From an instrumental viewpoint, Cottet et al. modified a commercial capillary electrophoresis instrument based on the differential measurement of the peak dispersion using the double detection of the sample zone. ${ }^{7}$

Amongst the employed capillary electrophoresis methods, capillary isoelectric focusing (CIEF), equipped with whole column imaging detection, is a relatively new, fast, automated, and simple technique. ${ }^{8}$ Direct monitoring of the diffusion process in real time, which is enabled by the application of whole-column imaging detection, greatly accelerates analysis. This simple method improves the temporal resolution of diffusion coefficient measurements. 
Although this approach is known as an effective way of providing diffusion measurements, there are inherent limitations within the method, such as the risk of protein precipitation at the isoelectric point (especially in higher concentrations of the sample and in the low ionic strength media), carrier ampholyte background absorption, and interaction issues. ${ }^{9,10}$ In the present work, imaging plug flow (iPF) technique is introduced to take advantage of wholecolumn imaging detection while addressing the drawbacks associated with CIEF method. In the iPF experiment, a commercial cartridge was employed, while the procedure was similar to the on-line pre-concentration method used by Yang et al. ${ }^{11}$

The fast Fourier transformation (FFT) approach reduces the effect of non-ideal characteristic shapes on the estimation of $\mathrm{D}$, as a typical error associated with analysis conducted in the time domain. The assumption of Gaussian peak shape for the diffusing signals restricts the calculations, while Fourier analysis offers a non-parametric measurement of diffusion coefficient, which is not dependent on peak shape. The successful application of the FFT approach in determination of diffusion coefficients has been already proven with fluorescence recovery after photo-bleaching, ${ }^{12}$ light-scattering, ${ }^{13}$ and chip-based electrophoresis ${ }^{14}$ techniques.

In this paper, we have provided an overview of the fast Fourier transformation approach in comparison with time domain analysis for the determination of diffusion coefficients. First, various peak shapes are simulated in order to further explore the shape-independent feature of this technique. Next, optimization of the method and investigation of method robustness in the presence of noise are discussed. Lastly, the FFT approach is experimentally validated by analyzing and comparing whole-column imaging detection CIEF and iPF results in the determination of diffusion coefficients. 


\section{Methods and Materials}

\section{Theory}

In order to extract the diffusion coefficient, the spatial decay of signals can be either studied in the time domain or carried to the frequency domain. The principles of both conventional time domain calculations and the rarely-used Fourier analysis are summarized here and further discussed in Supporting Information.

\section{Time Domain Approach}

Capillary electrophoresis data are originally time-based, and calculations are typically performed on the basis of the Gaussian shape assumption. ${ }^{15}$ While advanced software and electronic integrators are available to measure variances $\left(\sigma^{2}\right)$, the full width at half maximum or $\mathrm{W}_{(1 / 2)}$ is a common approach to approximate the variance. According to the Einstein equation, the diffusion coefficient is related to peak area at its corresponding time:

$$
\sigma^{2}=2 \mathrm{Dt}
$$

The concentration profile is fitted to a Gaussian function and the variance is approximated from $\mathrm{W}_{(1 / 2)}$. Finally, the peak variance is plotted against the diffusion time (t) and $\mathrm{D}$ is simply calculated from the slope of this linear curve.

\section{Frequency Domain Approach}

The diffusion coefficient value can be estimated from the spatial frequency component decay as diffusion proceeds. Fick's second law shows the rate at which concentrations are changing at any given point in space. In other words, this partial differential equation predicts how diffusion causes concentration to change over time. 


$$
\frac{\partial \mathrm{C}(\mathrm{x}, \mathrm{t})}{\partial \mathrm{t}}=\mathrm{D} \frac{\partial^{2} \mathrm{C}(\mathrm{x}, \mathrm{t})}{\partial \mathrm{x}^{2}}
$$

The Fourier transformation solution to the above differential equation can be calculated as:

$$
\mathrm{F} \mathrm{T}[\mathrm{C}(\mathrm{x}, \mathrm{t})]=\exp \left[-\mathrm{D} \omega^{2} \mathrm{t}\right] \mathrm{F} \mathrm{T}\left[\mathrm{C}\left(\mathrm{x}, \mathrm{t}_{0}\right)\right]
$$

(3) According to this equation, the diffusion process can be described as an exponential term, which filters out the high-frequency components. The diffusion coefficient can be extracted by rearranging Equation 3, which results in a new term, Q, with a linear dependence on time.

$$
\begin{aligned}
& \mathrm{Q}=-\ln \underline{\mathrm{F}} \underline{\mathrm{T}}\left(\mathrm{C}_{\mathrm{i}}\right)=\mathrm{Dt} \\
& / \omega^{2} \quad \mathrm{FT}\left(\mathrm{C}_{0}\right)
\end{aligned}
$$

where $\mathrm{C}_{0}$ and $\mathrm{C}_{\mathrm{i}}$ denote the concentration profiles at the initial and given times, and $\omega$ stands for the corresponding spatial divisions in the frequency domain. Knowing the FFT absolute values of the decaying signals at each time, the $\mathrm{Q}$ versus $\mathrm{t}$ plot provides a linear curve with a slope that corresponds to the diffusion coefficient from the frequency domain.

\section{Experimental Section}

Diffusion coefficient measurements are performed in real time with whole column imaging detection. The instrument runs at ambient temperature, while the variation is assumed to be identical between runs. Joule heating in both CIEF and iPF methods is insignificant due to low generated current at diffusion stage, with a maxima of $20 \mu \mathrm{A}$. Two different experimental approaches are employed for sample stacking under an electric field, then the relaxation period is recorded. 


\section{Chemicals and Sample Preparation}

Pharmalytes (pH 3-10), poly vinyl pyrrolidone (PVP) and other proteins were purchased from Sigma. The electrolytes were phosphoric acid and sodium hydroxide $100 \mathrm{mM}$ for the CIEF experiment, and phosphate buffer $(\mathrm{pH}=2.4$, and concentration: $25 \mathrm{mM}, 100 \mathrm{mM})$ for the iPF experiment. The protein samples were $0.25-0.5(\mathrm{mg} / \mathrm{mL})$, containing $2 \%$ pharmalytes and 0.5\% PVP. Ultrapure water (18 M $\Omega$ ) from Barnstead/Thermolyne system (Dubuque, IA, USA) was used for the preparation of all samples. The samples were filtered with a 0.2

$\mu \mathrm{m}$ pore-size membrane. The cartridge was conditioned with a $0.5 \%(\mathrm{w} / \mathrm{v})$ PVP solution.

\section{Methodology and Instrumentation}

All experiments were conducted in a commercial iCE280 analyser (Convergent Bioscience (now Protein Simple), Toronto, Canada). The instrument was equipped with a UV whole column imaging detector that was carried out at $280 \mathrm{~nm}$. For the plug flow analysis, a high voltage power supply (Stanford Research Systems, INC. Model PS350) was employed.

\section{Capillary Isoelectric Focusing (CIEF)}

The protein sample was hydrodynamically injected to fill the separation column. The initial applied voltage was set at $0.5 \mathrm{kV}$ for 3 min, increased to $3 \mathrm{kV}$ until the focused sample became stable, and then the voltage was turned off (usually t 10 min total run time). The diffusion process commenced as soon as the electric field was disconnected, and the diffusing protein concentration profiles were recorded at desired time intervals.

Imaging Plug Flow (iPF)

Field-amplified sample stacking ${ }^{16,17}$ is a simple stacking technique that has been developed to address the sensitivity issue in capillary electrophoresis. It has been adopted here for iPF and is represented schematically in in Figure S-14. The capillary (commercial cartridge) was filled with water and the protein sample in a low-conductivity matrix (lower concentration than the run buffer) was slightly concentrated under the membrane by electrokinetic injection ( $3 \mathrm{kV}$ for $1-2 \mathrm{~min}$ ). The plug was pushed into the middle of the capillary under the 
electeric field $(0.5 \mathrm{kV})$. The voltage was then disconnected, and the decaying signals were recorded by the whole column imaging detection system.

\section{Simulation Results and Discussion}

The shape independent characteristic of the FFT approach was studied through simulations in MatLab (R2013a) software. The effect of spatial and temporal parameters on determination of the diffusion coefficient and the noise tolerance of the technique were investigated.

Various peak shape models and signal processing techniques have been reviewed for capillary electrophoresis, ${ }^{18,19}$ including signal de-noising and baseline correction, both of which are common in electrophoretic separations. ${ }^{20}$

\section{Different Concentration Distributions}

The diffusion processes in conventional CIEF and the proposed iPF technique are modeled by Gaussian (G) and Boxcar (BC) functions, respectively, while the mathematical function Exponentially Modified Gaussian (EMG) represents common electropherogram imperfections such as tailing and fronting (Figure S-6 to S-10). Deviation from the expected symmetrical Gaussian shape in the electrophoretic peaks imposes an error in D calculations. ${ }^{21}$ For example, peak tailing underestimates the diffusion coefficient, while sample overload and slow migration in highly concentrated samples lead to overestimation. ${ }^{22}$ Many problems associated with the EMG model in the time domain can be addressed in the frequency domain. ${ }^{24}$

Diffusion acts as a filter that cuts high frequencies, hence signals with different peak shapes affect the calculations in the frequency domain, based on their frequency components. In the beginning, the sample plug is in the highest concentration gradient; thus, distinct borders create higher frequencies. As time passes, the peak shapes become broader and lose high their frequency components. Although the FFT approach is not restricted to any shape assumption, it depends on the shape of the signal, which creates different frequency 
components. Larger plug lengths lead to a delay in the transition from a Boxcar to a Gaussian profile of smearing peaks, and hence, the signal has high frequencies even at longer times. The larger the injection volume, the more deviation is expected from the Gaussian peak shape, whereas smaller injection volumes lead to poorer repeatability. ${ }^{23}$

\section{Optimization}

The spatial decay of the sample plug over time is considered a diffusion process. Hence, the Gaussian function is used to optimize both spatial and temporal aspects of the analysis. The assigned initial values are adopted from experimental conditions (instrument iCE280):

- Diffusion coefficient, moderate molecular weight proteins $\mathrm{D}=10^{-6}\left(\frac{\mathrm{cm}}{\mathrm{s}} 2\right)$.

- Resolution, the camera pixels over the column length, $\mathrm{p}=0.002\left(\frac{\mathrm{cm}}{\mathrm{pix}}\right)$.

- Initial time $\left(\mathrm{t}_{0}=0\right)$, with time interval $\left(\mathrm{t}_{\mathrm{i}}=30\left(\frac{\mathrm{sec}}{\mathrm{scan}}\right)\right)$ acquisition rate.

\section{Spatial Perspective}

In addition to choosing appropriate spatial resolution, it is crucial to select the working region properly.

Selected Region Monitoring (SRM) An effective sampling length that only brackets the desired peak will improve results by removing irrelevant information such as noisy baseline and spikes. Selecting the sampling region in the frequency domain is defined as convolving a boxcar window to the Gaussian signal; if the peak is not fairly preserved, the truncated signal creates false high frequencies that affect the estimation severely.

Table 1: Effect of SRM on determination of D, $\left(D=1 \times 10^{-6}\left(\mathrm{~cm}^{2} / \mathrm{s}\right), \mathrm{t}_{0}=30 \mathrm{~s}, \mathrm{t}_{\mathrm{i}}=30\right.$ $\mathrm{s}$,

$\mathrm{t}_{\mathrm{t}}=30 \mathrm{~min}, \mathrm{p}=0.002(\mathrm{~cm} / \mathrm{pix})$, signal to noise ratio(SNR) $\left.=3, \mathrm{n}=1000\right)$.

\begin{tabular}{llllll} 
Selected Region & $\pm \sigma$ & $\pm 2 \sigma$ & $\pm 5 \sigma$ & $\pm 10 \sigma$ & Whole channel \\
\hline X-limits (cm) & \pm 0.035 & \pm 0.07 & \pm 0.175 & \pm 0.35 & \pm 2.5 \\
Error\% $_{\text {(F req.) }}$ & 64.89 & 26.58 & 0.56 & 0.59 & 1.15 \\
\hline
\end{tabular}


Different D values are associated with different $\sigma$ regions. Using Einstien equation (Eq. 1 ), the $\sigma$ is calculated at the final time where the peak is in its broadest shape. The error associated with $\pm(5-10) \sigma$ is lower than $\% 0.6$, as shown in Table 1 . Working within the

$\pm 10 \sigma$ range is determined as the optimum peak region in order to eliminate artifacts while preserving the peak.

Resolution The current instrument provides a high resolution of $0.00 \operatorname{pix}_{\mathrm{ix}}(\underline{\mathrm{cm}})$. To find the resolution bottleneck, the narrowest variance of the diffusing plugs $\left(\sigma_{0}\right)^{2}$ can be calculated at the initial time. In practice, the sample plug is not an infinitely sharp zone, and in experiments, $t_{0}=0$ denotes the immediate scan after electric field removal. In simulation, the initial time is set to the first scan interval $t_{0}=30 \mathrm{~s}$ because the delta Dirac function generates a line which is smaller than the digitized pixel size.

Table 2: Effect of resolution on determination of $D,\left(D=1 \times 10^{-6}\left(\mathrm{~cm}^{2} / \mathrm{s}\right), \mathrm{t}_{0}=30 \mathrm{~s}, \mathrm{t}_{\mathrm{i}}=\right.$ 30

$\mathrm{s}, \mathrm{t}_{\mathrm{t}}=30$ min, Selected Region $\left.= \pm 10 \sigma, \mathrm{SNR}=3, \mathrm{n}=1000\right)$.

\begin{tabular}{llllllll} 
Division (cm/pix) & 0.01 & 0.007 & 0.0035 & 0.002 & 0.001 & 0.0002 & 0.0001 \\
\hline No. Data Points & 85 & 120 & 240 & 420 & 840 & 4200 & 8400 \\
\%Error $_{\text {(F req.) }}$ & 1.66 & 1.32 & 0.83 & 0.61 & 0.38 & 0.16 & 0.10 \\
\hline
\end{tabular}

High frequency details can be enhanced by sharpening the edges through an increase in resolution, but random noise also has a high spatial frequency characteristic that can cause an artifact. As shown in Table 2, the frequency domain analysis worked efficiently even at low resolutions. Hence, simulation results suggested that the resolution of the instrument was sufficient for this purpose, and little improvement in the results did not justify the expense of a higher resolution camera.

\section{Temporal Perspective}

It is important to investigate how long and at what frequency the diffusing signals should be recorded. Short analysis time is desirable in favour of high throughput analysis; however, a longer period would provide the averaging advantage which results in the precision and 
accuracy improvement. ${ }^{25}$ The nature of diffusing analytes needs to be taken into account for optimization of diffusion coefficient calculations. For instance, amino acids and smaller peptides with fast diffusive behavior have shorter optimum total time, while the diffusion process of big proteins with small diffusion coefficients need to be monitored for a longer period.

\section{Diffusion Process Duration}

At a given noise level, the slope of the linear curve varies at different lengths of analysis time. Three time windows of 10, 30, and 60 minutes are studied as short, medium, and long periods.

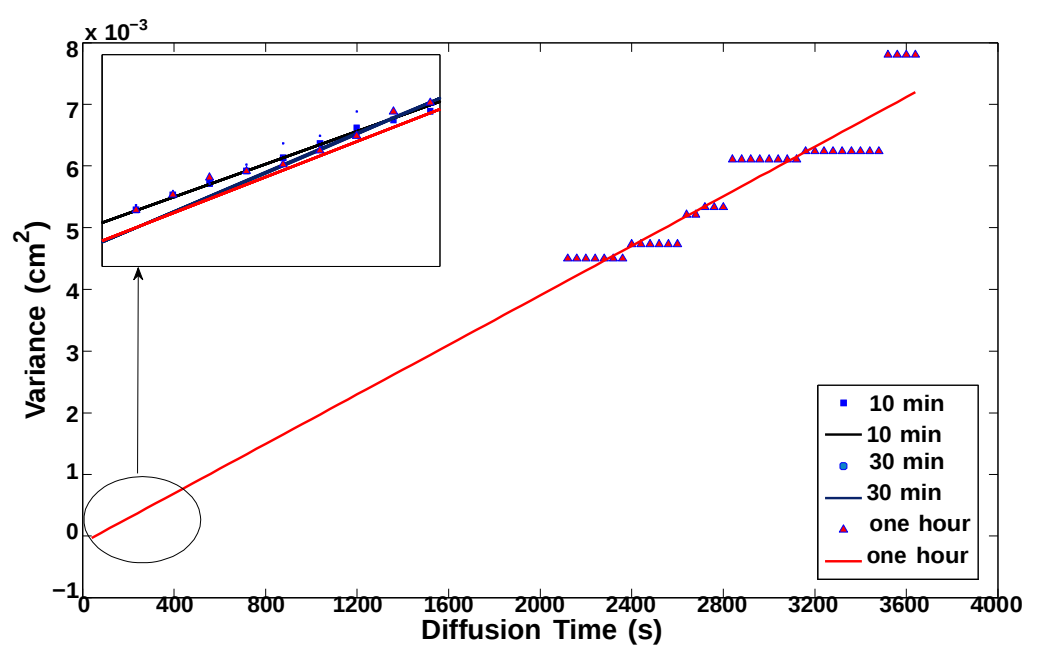

Figure 1: Determination of diffusion coefficient at 10,30 , and $60 \mathrm{~min}$ total time, $\left(\mathrm{D}=1 \times 10^{-6}\right.$ $\mathrm{cm}^{2} / \mathrm{s}, \mathrm{t}_{0}=30 \mathrm{~s}, \mathrm{t}_{\mathrm{i}}=30 \mathrm{~s}, \mathrm{p}=0.001$ (cm/pix), SNR=15, $\left.\mathrm{n}=1000\right)$.

As it is demonstrated in Figure 1 along with the results in Table S-3, a slight difference in the slope of the curves affects the results severely, revealing the sensitivity of the measurement toward total time. The effective diffusion time duration depends on the broadening sample plug characteristic. For example, small solutes with large diffusion coefficients need to be recorded with consecutive scans at short intervals that convey the most useful information on its diffusion. The weaker signal to noise ratio at a longer time period made the collected data less reliable, therefore the non-linearity increased by taking them into account. At higher 
noise levels this effect is more significant (see Supporting Information). In the studied system, a 30-min total time was determined to be the optimum period for the applied conditions, imposing the least error at the expense of overall non-linearity.

Data Acquisition Rate The sampling rate was investigated through two viewpoints of averaging different number of data points at a given total time and also considering the reliability of the collected data. For example, amino acids with large $\mathrm{D}$ values have fast decay rates, and when analyzing them, their signal is soon buried in noise. Hence, short sampling times with fast scan rates are required for the analysis of proteins with large D values.

The simulation results in the scatter plot, Figure S-12, demonstrate that the scan intervals did not significantly affect the estimation, and the error percent remained below one percent even at low temporal resolution of 5 minutes. As such, it is clear that a compromise was reached between the averaging advantage and the data reliability.

\section{Technique Robustness}

Amongst the parameters that would affect the determination of the diffusion coefficient, noise is an inherent feature of any instrumental technique.

Table 3: Technique robustness toward noise, error percent in estimation of $\mathrm{D}$ in both domains, $\mathrm{D}=1 \times 10^{-6}\left(\mathrm{~cm}^{2} / \mathrm{s}\right), \mathrm{t}=30 \mathrm{~min}, \mathrm{n}=1000$.

\begin{tabular}{|c|c|c|c|c|c|}
\hline SNR & 1 & 3 & 5 & 15 & 50 \\
\hline$\% \mathrm{E}_{\mathrm{E}}$ & $\begin{array}{r}36.61 \\
0.69\end{array}$ & $\begin{array}{r}24.30 \\
0.56\end{array}$ & $\begin{array}{c}18.51 \\
0.41\end{array}$ & $\begin{array}{l}5.57 \\
0.14\end{array}$ & $\begin{array}{l}4.57 \\
2.48 \times\end{array}$ \\
\hline
\end{tabular}

As can be concluded from Table 3 , the use of the $\mathrm{W}_{1 / 2}$ approach for the determination of diffusion coefficients can be significantly affected by noise in comparison to the frequency domain, which is highly tolerant against noise. The relatively noise-free $(\mathrm{SNR}=50)$ data provided by the frequency domain has relative standard deviation (RSD) equal to 2.03 and 
0.11 for time and frequency approaches respectively. However, noisy data in the time domain significantly lowers precision ( $R S D=20.43)$ more than the frequency domain $(\mathrm{RSD}=1.86)$.

\section{Experimental Results and Discussion}

As capillary electrophoresis peaks are not perfectly Gaussian, this imposes an error in the time domain calculations. As proven by the simulations, Fourier analysis is an alternative approach that can be applied to address this drawback. This technique facilitates employment of various instrumental methods such as iPF regardless of the peak shape. In this section, a set of experiments is conducted in CIEF and iPF, followed by efficient data analysis in the Frequency domain. The shape independent feature of the frequency domain analysis is demonstrated by the analysis of proteins with different peak shapes.

\section{Accurate Determination of Diffusion Coefficient}

The diffusion coefficients of several proteins have been determined by CIEF, and analysed in both the time and frequency domains. The results are summarized in Table 4, and accuracy was investigated via literature assessment. However, D values are primarily dependent on the empirical measurement technique used for their determination; for example, in CIEF, they are collected at zero net charge (pI), whereas in iPF, the intermolecular electrostatic forces are present. As is clearly observed from the experimental results, accuracy in the de- termination of diffusion coefficients significantly improved with the FFT method. Acceptable reproducibility was obtained, with the RSD below $8 \%$ for three replicates.

The significance of the FFT approach is more apparent when peak shapes deviate strongly from Gaussian distribution. In Figure 2, myoglobin (the major isoform), represents a fairly Gaussian shape, whereas albumin deviates from normal distribution (Figure S-17). In contrast with the full width at half maximum method, which imposed significant error in time domain calculations, the FFT method provided acceptable results for the irregular shapes. 
Table 4: Determination of diffusion coefficient by CIEF method followed by frequency $\left(D_{\mathrm{F} \text { req. }}\right)$ and time $\left(\mathrm{D}_{\mathrm{T} \text { ime }}\right)$ domain analysis, ${ }^{*}\left(\times 10^{7} \mathrm{~s} / \mathrm{cm}^{2}\right)$. Data are modelled by a sin- gle Gaussian function, in the curve fitting method.

\begin{tabular}{|c|c|c|c|c|c|c|c|c|}
\hline \multirow{2}{*}{ Protein } & & \multicolumn{2}{|c|}{${ }^{*} \mathrm{D}_{\mathrm{T} \text { ime }}$} & & \multicolumn{3}{|c|}{ \%Error } & \multirow{2}{*}{$\begin{array}{l}\text { Ref. } \\
26\end{array}$} \\
\hline & & FWHM & Fitting & & Freq. & FWHM & Fitting & \\
\hline Angiotensin & 25.39 & $29.60^{[8]}$ & 23.68 & 25.25 & 0.55 & 17.22 & 6.22 & 27 \\
\hline$\beta$-lactoglobulin $A$ & 6.90 & 9.32 & 9.29 & 7.38 & 6.50 & 26.29 & 25.88 & 28 \\
\hline$\beta$-lactoglobulin B & 3.12 & 3.96 & 2.60 & 3.14 & 0.63 & 26.11 & 17.19 & 29 \\
\hline Albumin (BSA) & 5.14 & $7.75^{[8]}$ & 2.86 & 5.90 & 12.88 & 31.36 & 51.41 & 29 \\
\hline Carbonic Anhydrase I & 10.57 & $9.36^{\left[{ }^{8}\right]}$ & 7.66 & 10.66 & 0.84 & 12.20 & 28.11 & 29 \\
\hline Myoglobin (Horse Heart) & 11.20 & 12.50 & 12.37 & 11.30 & 0.88 & 10.62 & 9.46 & \\
\hline
\end{tabular}

In Table4, for the proteins with a relatively Gaussian shape, such as angiotensin and myoglobin estimation of D by fitting method works better than by FWHM. In the case of $\beta$-lactoglobulin, although the profile was Gaussian in shape, due to the short measurement time there were no significant improvement in the estimation of D by curve fitting method. For BSA and carbonic anhydrase the peak shapes deviate from single Gaussian function model; hence,a Gaussian Multiple-term (GMT) model was fitted to the data, improving the estimated D significantly (Table S-1). The corresponding figures and GMT fitting results are discussed in the Supplementary Information.

\section{Whole Column Imaging Detection of Plug Flow vs. CIEF}

The sample concentration profile is influenced by both the experimental technique employed, and the nature of the analyte itself. For instance, in CIEF, the desired analyte was focused in a narrow band, which was defined by a Gaussian function, whereas in iPF, a sample plug was accumulated under the membrane by an electrokinetic injection, producing a boxcar-like profile. In the literature, the diffusion coefficient of the same compound is often reported with different values in accordance with the measuring procedure used. Table 5 provides results from iPF and CIEF methods on myoglobin and BSA samples. Since imaging plug flow has the same mechanism as capillary zone electrophoresis, interactions between sample ions will be taken into account for the estimation of $\mathrm{D}$, unlike with CIEF, where the measurements 
are corglucted at a zero net charge.

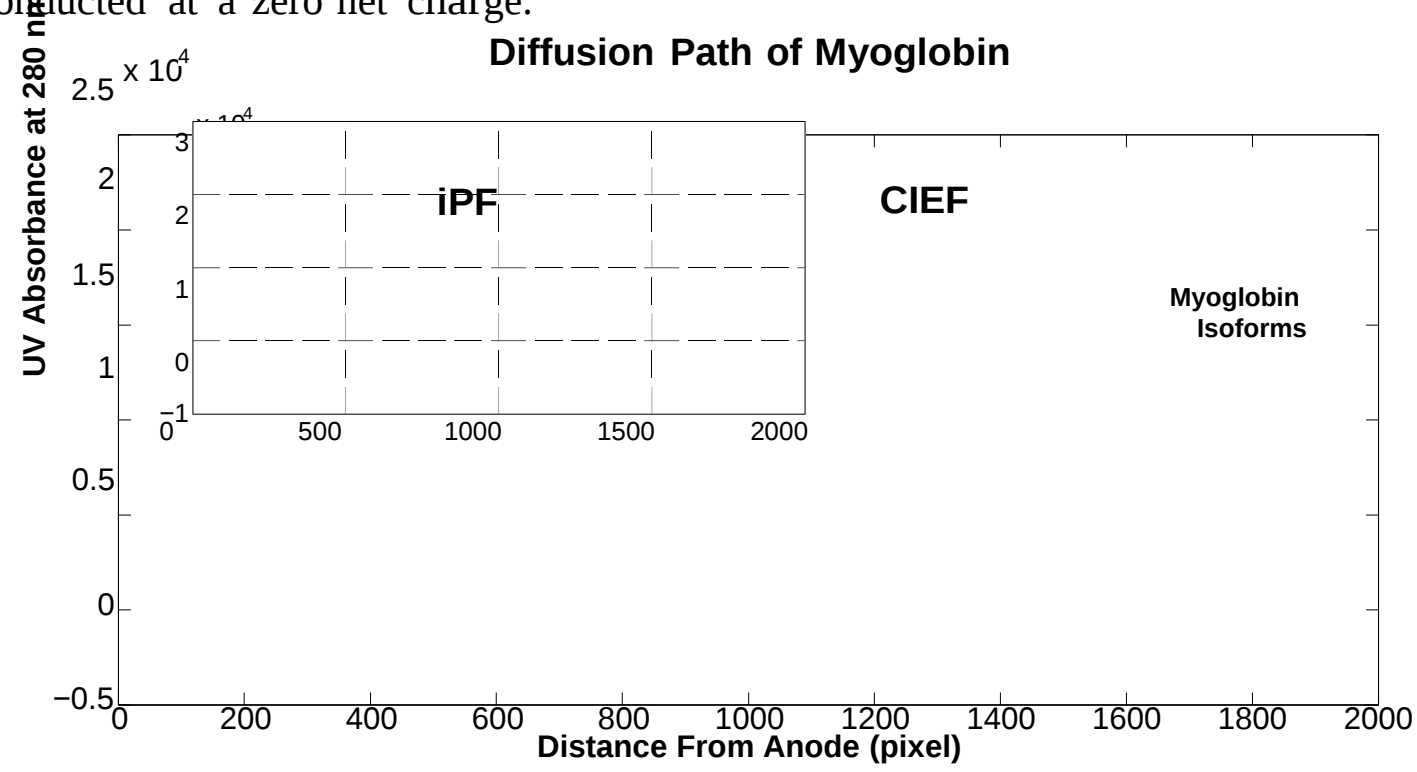

Figure 2: Demonstration of myoglobin iPF and CIEF diffusion profiles; stacking the sample $(0.35 \mathrm{mg} / \mathrm{ml}$ in $25 \mathrm{mM}$ buffer $)$ with run buffer $100 \mathrm{mM}, \mathrm{pH}=2.4$ at $3 \mathrm{kV}$ for $1 \mathrm{~min}$, then moving the sample plug by $0.5 \mathrm{kV}$, CIEF: the protein $0.35 \mathrm{mg} / \mathrm{ml}$ containing $2 \% \mathrm{pH}$ gradient $3-10$ under voltage gradient $0.5 \mathrm{kV}$ for $3 \mathrm{~min}$ and $3 \mathrm{kV}$ for $7 \mathrm{~min}$. After moving/focusing step, the electric field is removed and the diffusion pattern is recorded.

The change in the sample plug molecular weight reflects in the diffusion pattern, and can be monitored by iPF method. In the iPF inset in Figure 2, the myoglobin plug contains both isoforms, and the diffusion coefficient is an overall estimation of minor and major isoforms' presence. The minor isoform of myoglobin was determined to be $16.18 \times 10^{-7}\left(\mathrm{~cm}^{2} / \mathrm{s}\right)$ by CIEF. The diffusion coefficients associated with the major isoform and overall are presented in Table 5.

The results from iPF and CIEF show comparable accuracy; however, CIEF shows better precision. The plug flow experiment was designed for simple and fast measurement of diffusion coefficients at desired $\mathrm{pH}$ values. The reproducibility of the iPF results can be improved by modification of the cartridge, e.g. the T-shape injection configuration. The stacking step can also be preceded by an extra buffer wash to sweep away residuals, thus narrowing the plug and preventing tailing problem.

From a methodological perspective, the plug flow technique has several intrinsic advan- 
tages over the CIEF method, for example iPF eliminates problems associated with CIEF such as precipitation at the isoelectric point (pI) and carrier ampholyte background absorption. In addition, iPF is not limited by the isoelectric point of the species for the applied $\mathrm{pH}$ range, which facilitates the selection of a desired $\mathrm{pH}$, e.g. simulating in vivo conditions. It is also considered a relatively faster analysis technique that skips the focusing period, and replaces it with shorter sample stacking and moving times. In addition, the technique has great potential for miniaturization due to the absence of separation and focusing steps.

Table 5: Determination of BSA and myoglobin diffusion coefficients by CIEF and iPF methods, analysed with FFT approach. Experimental conditions the same as Figure 2. The literature values for BSA and myoglobin are $5.90 \times 10^{-7}$ and $11.30 \times 10^{-7}$ from reference [ $\left.{ }^{29}\right]$.

\begin{tabular}{|c|c|c|c|c|c|c|}
\hline Mehod & $\mathrm{D}_{\mathrm{BSA}}\left(\mathrm{cm}^{2} / \mathrm{s}\right)$ & $\mathrm{D}_{\mathrm{M} \mathrm{b}}\left(\mathrm{cm}^{2} / \mathrm{s}\right)$ & BSA $(n=3){ }^{R S I}$ & Mb. $(n=6)$ & BSA Error & \\
\hline CIEF & $5.14 \times 10^{-7}$ & $11.20 \times 10^{-7}$ & 4.30 & 6.51 & 12.88 & \\
\hline iPF & $6.49 \times 10^{-7}$ & $9.11 \times 10^{0.88}$ & 13.84 & 14.60 & 10.03 & - \\
\hline
\end{tabular}

\section{Protein Mixture Analysis in Short Time}

Simultaneous measurement of diffusion coefficients from mixed samples has been reported as the electromigration-based diffusivity spectrometry method, obtained by modifying the capillary electrophoresis system. ${ }^{30}$ However, the iCE280 instrument is a fast, automated, and commercially available tool that can also provide high resolutions of upwards $0.03 \mathrm{pH}$ unit $^{31}$ for this purpose. In this study, diffusion coefficients of $\beta$-lactoglobulin variants were determined and the effect of total analysis time was investigated.

In this mixture, the two peaks were resolved by a small pI difference $(\Delta p I=0.2)$ that started combining several minutes after diffusion commenced, providing a short period to record the diffusion process for each individual isoform. The diffusion coefficients of $\beta$ lactoglobulin A and B were determined in triplicates by FFT method in three time intervals (Table S-4). The collected data became more scattered as the measurement time was increased. This high throughput analysis is an advantageous feature of the CIEF method, 
followed by FFT data processing. However, the drawback of analysing the closely adjacent peaks is shorter measurement time that deprives from averaging.

\section{Investigation of Protein's Stability}

Capillary isoelectric focusing with whole column imaging detection can be used in food and pharmaceutical industries as a cost-effective and fast tool for quality control and aggregation study. The diffusion coefficient measurement can be used for molecular weight determination and stability assessment. In the present research the stability or shelf-life of $\beta$-lactoglobulin A and B variants were successfully investigated by monitoring the changes in the diffusion rates in addition to the charge heterogeneities and shift in pI values (Figure S-16). The fine alterations of the charge observed via high resolution power of the technique. The CIEF data evidenced the aggregate formation in the protein with remaining at room temperature for a period of one month (Table S-5).

\section{Conclusion}

The fast Fourier transformation technique addresses drawbacks of time domain analysis such as Gaussian shape assumption and rectifying the noisy signals. Taking advantage of the dynamic imaging detection system, the diffusion process is monitored in real time, and spatial data is carried to the frequency domain for determination of the diffusion coefficient. Thus, FFT analysis empowers the CIEF technique for measuring the diffusion coefficient. Using various functions, the research proves that this approach is not restricted by any peak shape assumption as it is in the time domain. Another advantage of FFT analysis is to retrieve information from signals buried in noise due to producing different frequencies.

The employed FFT model was validated experimentally through CIEF and iPF techniques. Accurate diffusion coefficients extracted by the FFT approach reinforced the method efficiency. However, there is a potential problem of truncation error associated with tailing 
peaks, which affects calculations in the frequency domain and need further investigations.

An important application of this technique is the fast recognition of the aggregation, which is of pharmaceutical interest. The diffusion coefficient changes provide information on variations in molecular weight and the hydrodynamic radius. The CIEF and iPF equipped with whole column imaging detection, beside current analytical technologies can assist detection, characterization, and quantisation of aggregates in protein products.

\section{Acknowledgement}

The authors thank Dr. H. Yeganeh and Miss. H. Zarabadi, whom kindly offered invaluable advice on programming.

\section{Nomenclature}

$\mathrm{t}_{0} \quad$ Initial time

$\mathbf{t}_{\mathbf{i}} \quad$ Time interval

BC Boxcar

CIEF Capillary isoelectric focusing

D Diffusion coefficient

EMG Exponentially modified Gaussian

FFT Fast Fourier transformation

G Gaussian

iPF Imaging plug flow 

n Number of iterations
p Pixel size
RSD Relative standard deviation
SNR Signal to noise ratio
SRM Selected region monitoring
WCID Whole column imaging detection

\section{Supporting Information Available}

This material is available free of charge via the Internet at http://pubs.acs.org/.

\section{References}

(1) Taylor, G. Proc. R. Soc. A 1953, 219, 186-203.

(2) Aris, R. Proc. R. Soc. A 1956, 235, 67-77.

(3) Bello, M. S.; Rezzonico, R.; Righetti, P. G. Science (N.Y.) 1994, 266, 773-6.

(4) Walbroehl, Y.; Jorgenson, J. W. J. Microcolumn Sep. 1989, 1, 41-45.

(5) Jin, W.; Chen, H. Chromatographia 2000, 52, 17-21.

(6) Sharma, U.; Gleason, N. J.; Carbeck, J. D. Anal. Chem. 2005, 77, 806-13.

(7) Chamieh, J.; Oukacine, F.; Cottet, H. J. Chromatogr. A 2012, 1235, 174-7.

(8) Liu, Z.; Lemma, T.; Pawliszyn, J. J. Proteome Res. 2006, 5, 1246-51.

(9) Wu, J.; Pawliszyn, J. Anal. Chem. 1992, 64, 219-24.

(10) Righetti, P. G. J. Chromatogr. A 2004, 1037, 491-9. 
(11) Yang, B.; Zhang, F.; Tian, H.; Guan, Y. J. Chromatogr. A 2006, 1117, 214-8.

(12) Tsay, T. T.; Jacobson, K. A. Biophys. J. 1991, 60, 360-8.

(13) Berk, D. A.; Yuan, F.; Leunig, M.; Jain, R. K. Biophys. J. 1993, 65, 2428-36.

(14) Gosse, C.; Saux, T. L.; Allemand, J.; Croquette, V.; Berthoumieux, H. Anal. Chem. 2007, 79, 8222-31.

(15) Culbertson, C. T.; Jacobson, S. C.; Michael Ramsey, J. Talanta 2002, 56, 365-73.

(16) Osbourn, D. M.; Weiss, D. J.; Lunte, C. E. Electrophoresis 2000, 21, 2768-79.

(17) Giordano, B. C.; Burgi, D. S.; Hart, S. J.; Terray, A. Anal. Chim. Acta 2012, 718, $11-24$.

(18) Stewart, R.; Gideoni, I.; Zhu, Y. In Systems and Computational Biology-Bioinformatics and Computational Modeling; Yang, N.-S., Ed.; InTech, 2011; Chapter 15, pp 311-34.

(19) Gas, B.; Stedry, M.; Rizzi, a.; Kenndler, E. Electrophoresis 1995, 16, 958-67.

(20) Liu, B.-F.; Sera, Y.; Matsubara, N.; Otsuka, K.; Terabe, S. Electrophoresis 2003, 24, $3260-5$.

(21) Gebauer, P.; Boc, P. Anal. Chem. 1997, 69, 1557-63.

(22) Zou, M.; Han, Y.; Qi, L.; Chen, Y. Chin. Sci. Bull. 2007, 52, 3325-32.

(23) Ye, F.; Jensen, H.; Larsen, S. W.; Yaghmur, A.; Larsen, C.; Ø stergaard, J. J. Pharm. Biomed. Anal. 2012, 61, 176-83.

(24) Felinger, a.; Pap, T.; Inczédy, J. Talanta 1994, 41, 1119-26.

(25) Thetford, C. M. Ph.D. Thesis ; University of North Carolina, 2003.

(26) Behlke, J.; Ristau, O. Biochem. Soc. Trans. 1998, 26, 758-61. 
(27) Chu, B.; Yeh, A.; Chen, F. C.; Weiner, B. Biopolymers 1975, 14, 93-109.

(28) Chen, B.; Andreas, C.; David, R. Anal. Biochem. 1979, 96, 120-30.

(29) Smith, M. H. In Handbook of Biochemistry; 2nd,, Ed.; Sober,H. A., Ed: Chemical Rubber Company, Cleveland, 1970.

(30) Yang, S.; Zhang, Y.; Liao, T.; Guo, Z.; Chen, Y. Electrophoresis 2010, 31, 2949-56.

(31) Fang, X.; Tragas, C.; Wu, J.; Mao, Q.; Pawliszyn, J. Electrophoresis 1998, 19, 2290 5. 
Graphical TOC Entry

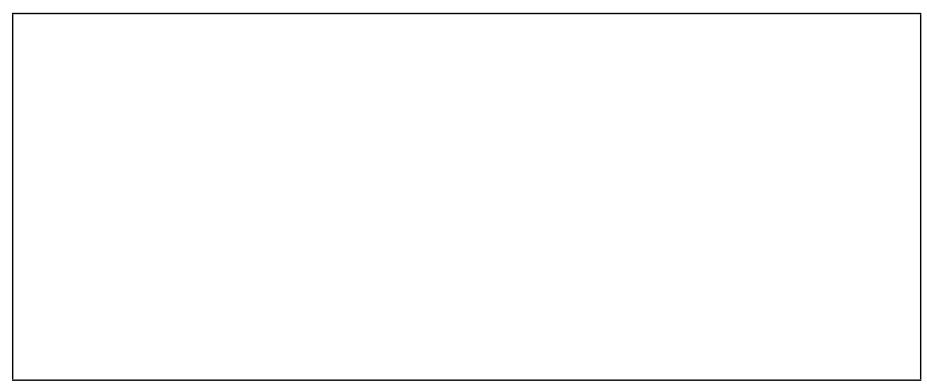

